\title{
Fluorescently-Labeled Estradiol Internalization and Membrane Trafficking in Live N-38 Neuronal Cells Visualized with Total Internal Reflection Fluorescence Microscopy
} Kassandra Kisler, Robert H Chow and Reymundo Dominguez*

Zilkha Neurogenetic Institute, Department of Physiology and Biophysics, Keck School of Medicine of University of Southern California, USA

\begin{abstract}
Estradiol is a steroid hormone that binds and activates estradiol receptors. Activation of these receptors is known to modulate neuronal physiology and provide neuroprotection, but it is not completely understood how estradiol mediates these actions on the nervous system. Activation of a sub-population of estradiol receptor- $\alpha(E R \alpha)$, originally identified as a nuclear protein, localizes to the plasma membrane and appears to be a critical step in neuroprotection against brain injury and disease. Previously we showed that estradiol stimulates the rapid and transient trafficking of plasma membrane ERa in primary hypothalamic neurons, and internalization of membrane-impermeant estradiol (E6BSAFITC) into cortical neuron endosomes in vitro. These findings support the concept that estradiol activates and downregulates plasma membrane $\mathrm{ER} \alpha$ by triggering endocytosis. Here, we use TIRFM (total internal reflection fluorescence microscopy) to image the trafficking of E6BSA-FITC, and GFP-labeled ERa, in live cells in real time. We show that activation of plasma membrane ERs by E6BSA-FITC result in internalization of the fluorescent ligand in live $\mathrm{N}-38$ neurons, an immortalized hypothalamic cell line. Pretreatment with ER antagonist ICI 182,780 decreased the number of E6BSA-FITC labeled puncta observed. We also observed in live N-38 neurons that E6BSA-FITC co-localized with FM4-64 and LysoTracker fluorescent dyes that label endosomes and lysosomes. Our results provide further evidence that plasma membrane $\mathrm{ER} \alpha$ activation results in endocytosis of the receptor.
\end{abstract}

Keywords: Estradiol; Estrogen receptor alpha; Plasma membrane; Trafficking; Endocytosis; E6BSA-FITC; Total Internal Reflection Fluorescence Microscopy (TIRFM); Endocytosis; Exocytosis; Live-cell

\section{Introduction}

Estradiol is a sex steroid hormone involved in central nervous system organization and activation [1]. Estradiol carries out its actions by activating estrogen receptors (ERs), of which there are two classes, $\mathrm{ER} \alpha$ and ER $\beta$. In the brain both ERs are expressed within the nucleus and cytoplasm, as well as localized to the plasma membrane of neurons and astrocytes [2]. Moreover, evidence suggests that other ER-like membrane receptors (e.g. GPR30, ER-X, mGq-ER) can also become activated upon estradiol binding [3,4].

ERs are part of a family of ligand-activated transcription factors. When intracellular ERs become active by ligand binding they form dimers that then travel to the nucleus and bind to DNA regions containing estrogen-sensitive promoter sequences [5]. In contrast to this genomic mechanism, studies have shown that ERs present at the plasma membrane can rapidly trigger the activation of ion channels and second messenger systems, even in the presence of protein synthesis inhibitors, by a mechanism that is not completely understood [6-9].

Membrane receptors as a group are regulated through transcription and by trafficking. Removal of receptors from the cell membrane via internalization is a well-characterized mechanism of receptor signal termination [10]. Internalized receptors are transported to endosomes where the ligand is released from the receptor and is then sorted for either recycling back to the membrane or degradation [11]. Trafficking of membrane ERs has thus far been difficult to observe, however there is compelling evidence that activated membrane ERs can be inserted into and internalized from the plasma membranes in primary hypothalamic neurons and astrocytes [12-14].

To study receptor trafficking investigators have used biochemical and histochemical techniques to track the localization of the receptor or a receptor-specific ligand. Using cell surface biotinylation and western blotting we previously found that estradiol treatment for 30 minutes significantly increased the levels of full-length ERa and the $\mathrm{ER} \alpha \Delta 4$ splice variant present on the plasma membrane of primary native hypothalamic neurons and N-38 neurons $[4,14]$. N-38 neurons are immortalized neuropeptide-Y secreting neurons derived from the arcuate nucleus of the hypothalamus of mice and they serve as an accepted model for studies of mechanisms involved in estradiol signaling $[4,15,16]$. While these biochemical studies have shown that active fulllength ERa is rapidly trafficked into and out of the plasma membrane, there have been few attempts to visualize this process, particularly in live neurons [17]. In the present study we use live N-38 neurons and total internal reflection fluorescence microscopy (TIRFM) to image the trafficking of the membrane impermeant ER-ligand E6BSA-FITC and image GFP-ER $\alpha$ near or at the cell surface in real time. For example, similar studies examining nicotinic acetylcholine receptor trafficking have used the receptor-specific ligand $\alpha$-bungarotoxin in real-time [1820].

TIRFM is ideal for studying estradiol stimulated trafficking of plasma membrane ER in living cells because it gives high-contrast, real-time images of fluorescent molecules on and just within the cell basal surface. TIRFM is an established method to image the basal surface, or "footprints," of cells adhered to a glass substrate without

*Corresponding author: Reymundo Dominguez, Zilkha Neurogenetic Institute 323, Keck School of Medicine of USC, Los Angeles, CA 90089-2821,USA, E-mail: reymundd@usc.edu

Received March 01, 2013; Accepted April 15, 2013; Published April 20, 2013

Citation: Kisler K, Chow RH, Dominguez R (2013) Fluorescently-Labeled Estradio Internalization and Membrane Trafficking in Live N-38 Neuronal Cells Visualized with Total Internal Reflection Fluorescence Microscopy. J Steroids Hormon Sci S12: 002. doi:10.4172/2157-7536.S12-002

Copyright: $\odot 2013$ Kisler K, et al. This is an open-access article distributed unde the terms of the Creative Commons Attribution License, which permits unrestricted use, distribution, and reproduction in any medium, provided the original author and source are credited. 
the background fluorescence contamination typical of other imaging methods, making it ideal for visualizing small fluorescent structures and kinetic events near the cell membrane such as trafficking, endocytosis, and exocytosis [e.g. 21-23]. TIRFM relies on light incident at the coverslip-cell interface at an angle greater than the critical to generate an evanescent (exponentially decaying) light wave perpendicular to the coverslip surface to excite flurophores. The light intensity drops off quickly, and is characterized by a "penetration depth" of $\sim 100 \mathrm{~nm}$ (Figures 1A and 1B). For these experiments, we used "through-theobjective" TIRFM [21], where excitation light is delivered to the cell through a high NA (numerical aperture) objective at an angle greater than the critical angle, and fluorescence excited is collected through the same objective (Figure 1A).

Here, particle analysis of TIRFM images of N-38 neurons showed E6BSA-FITC at the cell surface. The binding of E6BSA-FITC was blocked by pretreatment with the ER antagonist ICI 182,780. Furthermore, using sequential TIRFM images acquired in real time, we were able to track fluorescent E6BSA-FITC particles, and show that trafficking of the fluorescent ligand in and out of the plasma membrane occurred rapidly within seconds. Additionally, we could visualize GFP-labeled ERa in live cells, and show that the receptor, or internalized E6BSA-FITC, colocalized with fluorescently-labeled lysosomes, endosomes, and vesicles. Treatment of live N-38 neurons with E6BSA-FITC and TIRFM revealed that fluorescent ER-ligand could be visualized at a temporal and spatial scale not before reported for plasma membrane ER regulation.

\section{Material and Methods}

\section{$\mathrm{N}$-38 cultures and fluorescence assays}

Cultures were prepared from a frozen stock of N-38 neurons (Cellutions Biosystems, Burlington, ON, Canada) and cultured on custom coverslip-bottom (\#1 thickness) cell culture dishes. Cells were maintained in Dulbecco's Modified Eagle Medium (DMEM; 4.5 g/l glucose, L-glutamine, sodium pyruvate, $1 \%$ penicillin/streptomycin; Cellgro, Manassas, VA) with $10 \%$ heat inactivated fetal bovine serum (Invitrogen) at $37^{\circ} \mathrm{C}$ with $5 \% \mathrm{CO}_{2}$. One week prior to experimentation,

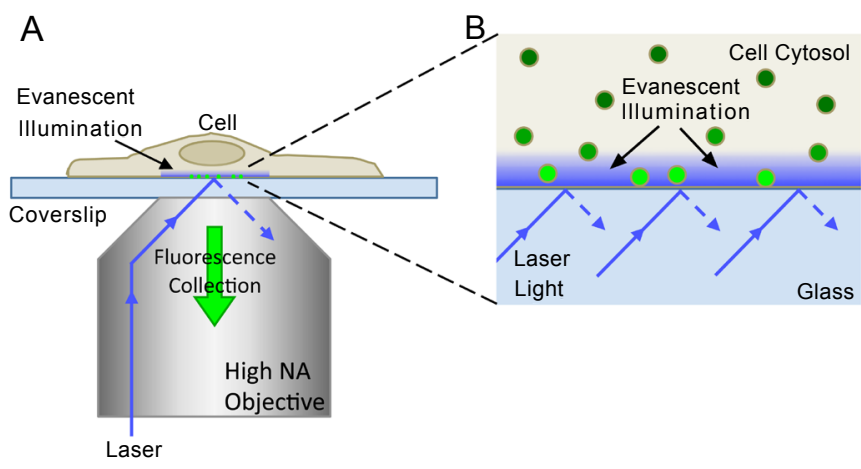

Figure 1: Through-the-objective TIRFM illumination and detection. (A) Laser light is directed through a high NA objective to the glass coverslip such that it is incident on interface at an angle greater than the critical angle, resulting in total internal reflection. This generates an evanescent wave that illuminates a short depth $(\sim 100 \mathrm{~nm})$ into the medium (cell). Fluorescence excited by evanescent illumination is collected through the same objective. (B) Magnification of the cell-coverslip interface region in (A). The evanescent light intensity decreases exponentially with depth. Only fluorophores within the depth of the evanescent illumination field are excited (bright green particles). Fluorescent particles far from the coverslip are not excited and thus do not fluoresce. Figures are not to scale. a stock solution of $10 \mathrm{mg} / \mathrm{ml}$ E6BSA-FITC was diluted with sterile $0.9 \%$ saline and prepared using an ultracentrifugation protocol to reduce free estradiol [24] and then stored at $-20^{\circ} \mathrm{C}$ until use. Control BSAFITC (Sigma) was prepared in a similar manner. On day 3 after plating, cultures were washed twice with phosphate buffer solution (PBS; 1 $\mathrm{mM} \mathrm{Ca} 2+, 1 \mathrm{mM} \mathrm{Mg} 2+, \mathrm{pH}$ 7.2) and then steroid-starved in DMEM with ITS (approximately $21 \mathrm{mg} / \mathrm{L}$ insulin, 19/L mg transferrin, $25 \mu \mathrm{g} / \mathrm{L}$ sodium selenite; Sigma) $24 \mathrm{~h}$ prior to any experimental treatments. On day 4 , cultures were treated for $30 \mathrm{~min}$ with $1 \mu \mathrm{g} / \mathrm{ml}$ fluoresceinlabeled $\beta$-estradiol-6-(O-carboxymethyl)oxime-bovine serum albumin (E6BSA-FITC, Sigma) or $1 \mu \mathrm{g} / \mathrm{ml}$ bovine serum albumin-fluorescein (BSA-FITC) in DMEM for imaging experiments. To antagonize estradiol receptor activation N-38 cultures were pretreated for $1 \mathrm{~h}$ with $1 \mu \mathrm{M}$ ICI 182,780 before cotreatment with E6BSA-FITC. For colocalization experiments, cells were treated with FM4-64 $(20 \mu \mathrm{g} / \mathrm{ml}$; Invitrogen) for $30 \mathrm{~min}$ after any other treatments. For LysoTracker red ( $25 \mathrm{nM}$; Invitrogen) cultures were treated for the last $5 \mathrm{~min}$ of experimental treatments. After treatments, the cells were washed and media replaced with warmed external solution (In mM: $140 \mathrm{NaCl}, 3$ $\mathrm{KCl}, 10$ HEPES, $1 \mathrm{MgCl}_{2}, 2 \mathrm{CaCl}_{2}, 15$ glucose, $\mathrm{pH} 7.3$ ) for imaging.

\section{cDNA plasmid transfection}

N-38 cultures were prepared as described above. GFP-ERa expression was induced by transfection with Lipofectamine 2000 (Invitrogen) and $200 \mathrm{ng}$ of cDNA plasmid encoding for human ERa conjugated to GFP [Addgene \#28230 pEGFP-C1-ERa; 25] following the manufacturer's protocol. Experiments were conducted $48 \mathrm{~h}$ after transfection.

\section{Total internal reflection fluorescence microscopy}

A custom-built laser TIRF microscope, based on an Olympus IX70 inverted microscope platform, was used to observe the cells [26]. Cells were illuminated with $488 \mathrm{~nm}$ light from an Argon laser (Coherent Inc, Santa Clara, CA) and/or $561 \mathrm{~nm}$ laser light through a high NA objective (a Plan-FLUAR 100X oil; NA 1.45, Ziess) utilizing a green/red dualbandpass dichroic (XF2046; Omega). Laser light was incident on the coverslip at an angle greater than the critical angle such that evanescent light was generated in a shallow layer $(\sim 100 \mathrm{~nm})$ above the coverslip. Fluorescence was collected through the same objective, passed through a Dual-View image splitter (Optical Insights) with $560 \mathrm{~nm}$ dichroic and HQ 515/30 $\mathrm{m}$ and D605/55 $\mathrm{m}$ (Chroma) emission filters, and imaged with a Cascade 512B EMCCD camera (Roper; operated with Metamorph 4.6, Universal Imaging). Images were acquired at room temperature.

\section{Image Acquisition and particle analysis}

Single images and sequential image series (sets of 500 frames at 4 $\mathrm{Hz}, 250 \mathrm{~ms}$ exposure per frame) were acquired for analyses. Analysis of images was performed using ImageJ64 (1.45e) software. For particle analysis (number FITC puncta, the area of FITC puncta coverage, and FITC puncta size), single images were thresholded (intermodes), then fluorescent puncta were found using ImageJ's buit-in routines. Particle sizes of less then 2 pixels $^{2}$ ( 1 pixel $=165 \mathrm{~nm}$ ) were considered artifacts and excluded from the analyses. Fluorescent particles observed during image sequences were analyzed using the MTrackJ (1.4.1) plug-in for ImageJ64 [27]. Single frame steps (from a sequence of 100 frames or $25 \mathrm{sec}$ ) along with local cursor snapping during tracking with a snap range of $13 \times 13$ pixels and bright centroid snap feature was used to track fluorescent puncta motion. Puncta were tracked for the duration they were visible in the image sequence. The Cairn Image-Splitter plug-in was used to align images for dual-color TIRFM experiments. 
Citation: Kisler K, Chow RH, Dominguez R (2013) Fluorescently-Labeled Estradiol Internalization and Membrane Trafficking in Live N-38 Neuronal Cells Visualized with Total Internal Reflection Fluorescence Microscopy. J Steroids Hormon Sci S12: 002. doi:10.4172/2157-7536.S12-002

Page 3 of 7

\section{Statistical analysis}

A one-way ANOVA followed by a Bonferroni post hoc test were used to compare the statistical significance between multiple experimental treatment groups. Significance values were calculated using Bonferroni Multiple Comparison Test unless otherwise stated. Data were analyzed using GraphPad Prism 4 software (GraphPad Software, La Jolla, CA), and significance level was set at $p<0.05$ for all experimental data sets.

\section{Results}

\section{TIRFM imaging of live E6BSA-FITC treated N-38 neurons}

TIRFM was used to visualize the binding and internalization of E6BSA-FITC to plasma membrane ERs (Figure 2A). After 30 minutes of treatment imaging showed the formation and aggregation of E6BSAFITC fluorescent molecules on the cell surface. Visually over time this appears as the formation of different sized spots of fluorescent label on the cell surface and cytoplasm $[28,29]$. BSA-FITC was also tested (bovine serum albumin-fluorescein isothio-cyanate) to determine if either BSA or FITC artifactually caused binding to the plasma membrane.

Particle analysis of fluorescent puncta ( 3 independent experiments with 4 or more cells imaged per condition) revealed that among the N-38 neurons treated with E6BSA-FITC 8 of 12 cells ( $67 \%)$ imaged contained several fluorescent puncta (Figure 2B), whereas among cells treated with BSA-FITC only 9 of 27 cells $(\sim 33 \%)$ contained

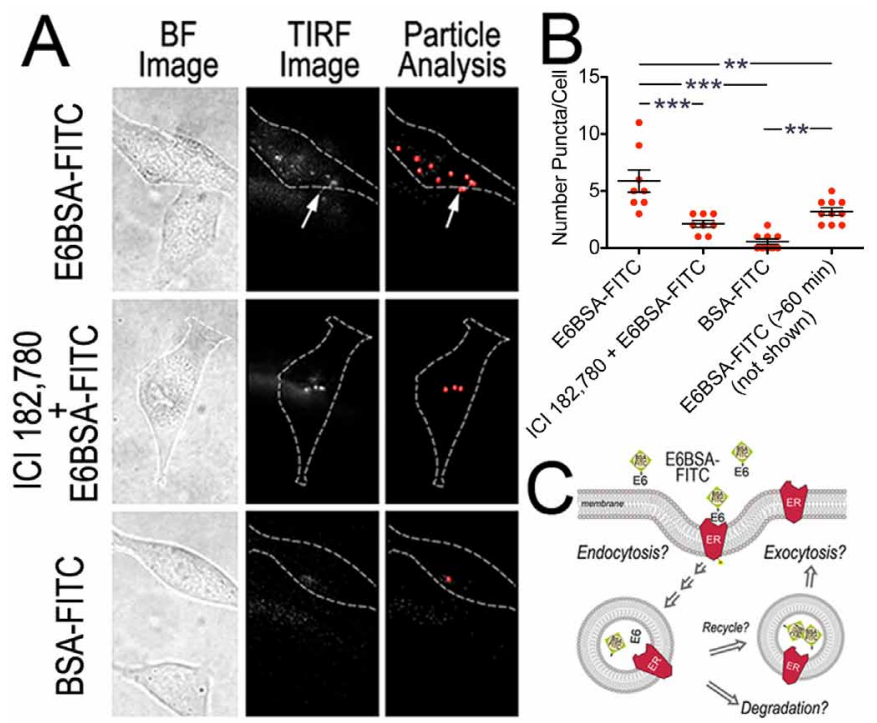

Figure 2: TIRFM analysis of fluorescent E6BSA-FITC molecules. (A) Brightfield (BF) and TIRFM images of steroid-starved N-38 cultures treated for 30 min with E6BSA-FITC, ICI 182,780 and E6BSA-FITC, or BSA-FITC. Dashed lines outline the cell body $(\mathrm{CB})$ perimeter; puncta found out-side the perimeter (arrow) were not computed. Images show E6BSA-FITC binds to membranes of specific cells, and is inhibited by $\mathrm{ICl} 182,780$. (B) Particle analysis (bar graph) of fluorescent puncta from TIRFM images revealed a significant number of fluorescent puncta present per cell for E6BSA-FITC treated cells compared to BSA-FITC treated cells. On average $5.875 \pm 0.972$ ( $n=8$ cells) fluorescent puncta were present in E6BSA-FITC treated cells compared to only $0.556 \pm$ $0.242 /$ cell for BSA-FITC $(1 \mu \mathrm{g} / \mathrm{ml} ; \mathrm{n}=9$ cells $)$. Pretreatment for $1 \mathrm{~h}$ with $1 \mu \mathrm{M} \mathrm{ICl}$ 182,780 reduced E6BSA-FITC binding $(2.125 \pm 0.295 ; n=8$ cells $)$ compared to E6BSA-FITC. The presence of E6BSA-FITC on the plasma membrane was transient; 60 min after the removal of E6BSA-FITC only $3.2 \pm 0.327$ ( $n=10$ cells) fluorescent puncta were present (TIRFM image not shown). (C) Schematic of E6BSA-FITC internalization and trafficking at the plasma membrane of N-38 neurons (Bonferroni post hoc test, ${ }^{* *} p<0.001,{ }^{* * *} p<0.0001$ ). puncta. Further, of those cells containing fluorescent puncta, there were significantly fewer puncta in BSA-FITC treated control cells than E6BSA-FITC treated cells (ANOVAp $<0.0001, \mathrm{~F}_{(3,31)}=18.19$ ). On average $5.875 \pm 0.972$ ( $\mathrm{n}=8$ cells) fluorescent puncta were present in E6BSA-FITC treated cells compared to only $0.556 \pm 0.242 /$ cell for BSAFITC $(1 \mu \mathrm{g} / \mathrm{ml} ; \mathrm{n}=9$ cells $)$. Continuous E6BSA-FITC treatment for $>60$ min resulted in a decrease number of E6BSA-FITC puncta present near the plasma membrane per cell to $3.2 \pm 0.327$ ( $n=10$ cells)

E6BSA-FITC specificity was examined by pre-treating cultures with the ER antagonist ICI 182,780 (ICI). We observed that ICI decreased the number of E6BSA-FITC fluorescent molecules present on the plasma membrane per N-38 neuron $(2.125 \pm 0.295 ; n=8$ cells $)$, but did not decrease the number of cells containing fluorescent molecules ( 8 of 11 cells; $73 \%$ ). Our TIRFM data suggest that E6BSA-FITC binds and becomes internalized (Figure 2C), presumably by binding to ERa proteins as well as other estradiol binding proteins present on the cell surface of N-38 neurons.

While it is not known exactly how many ER $\alpha$ receptors are expressed on the cell surface [30], less than 5\% of total ERa has been observed to localize to the plasma membrane of Chinese hamster ovary cells [31]. We chose to analyze fluorescent puncta smaller than or equal to $330 \mathrm{~nm}(2 \times 2$ pixels, calibrated at 1 pixel $=165 \mathrm{~nm})$. It is unclear whether the select fluorescent puncta we observed (220-330 $\mathrm{nm}$ ) represent single E6BSA-FITC molecules because the ER ligand contains more than one FITC label (3-5 mol FITC per mol of BSA). Complicating matters, each BSA molecule contains several estradiol molecules that may not bind (thus we use molar excess levels of E6BSAFITC) and that may bind to one or more ERs [5-10 mol estradiol per mol of BSA; 24,32,33].

\section{TIRFM shows GFP-ER $\alpha$ associated with endosomes, lysosomes, and vesicles}

In a separate set of experiments, N-38 neurons expressing human $\mathrm{ER} \alpha$ linked to green fluorescent protein (GFP-ER $\alpha$ ) colocalized with puncta labeled with either FM4-64 (membrane marker) or Lysotracker Red (Figures 3A and 3B). For comparison, E6BSA-FITC treated cells were co-labeled with FM4-64 (Figure 3C). FM4-64 is a lipophilic styryl dye that becomes fluorescent upon insertion into the plasma membrane and is routinely used for tracking membranes endocytosed from the plasma membrane [21,34]. FM4-64 belongs to a class of cellimpermeable fluorescent labels (FM dyes) that intercalates into the outer leaflet of the plasma membrane. In order for the label to appear inside the cell, the membrane to which it is bound must be internalized (endocytosed) [35-37]. LysoTracker Red is a fluorescent compound that permeates the plasma membranes of live cells and accumulates in low $\mathrm{pH}$ compartments, making it ideal for identifying acidic organelles [38] such as lysosomes and exocytic vesicles. Together our findings strongly suggest that estradiol treatment results in the endocytosis of plasma membrane ERs in N-38 neurons.

\section{Tracking the motion of E6BSA-FITC fluorescent molecules with TIRFM}

The tracking of fluorescent E6BSA-FITC puncta, using sequential TIRFM image frames, uncovered that E6BSA-FITC is rapidly (within seconds) trafficked not only inward, but also toward the cell surface, as well as laterally (Figures $4 \mathrm{~A}$ and $4 \mathrm{~B}$ ). We classified observed puncta based on their movement characteristics and changes in fluorescence intensity. Many of the E6BSA-FITC puncta observed, which we designated "Stationary Puncta," did not move appreciably 


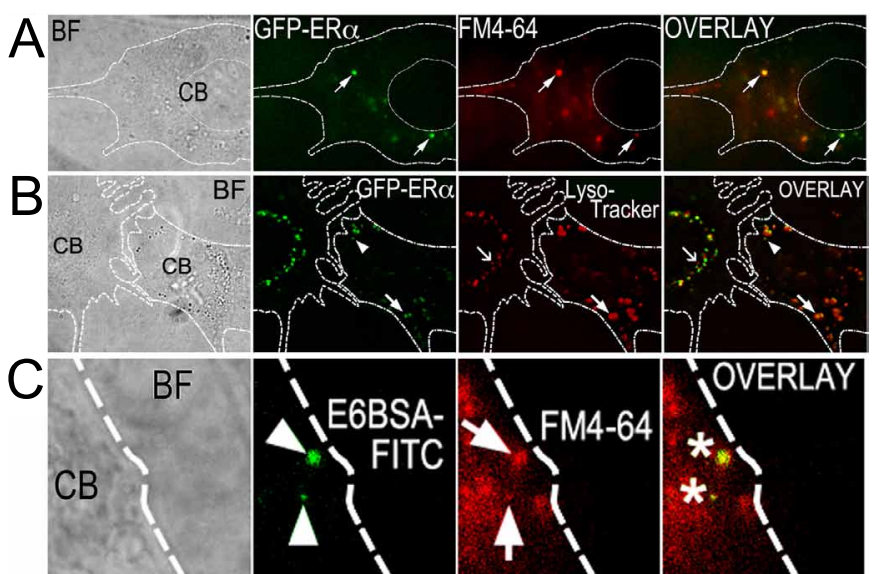

Figure 3: GFP-ERa colocalize with fluorescent endocytosis markers and E6BSA-FITC in TIRFM. (A,B) GFP-ER $\alpha$ are present on and around the plasma membrane of N-38 neurons. Overlay of FM4-64 (A) or Lysotraker Red (B) fluorescence reveals co-localization of GFP-ER $\alpha$ with vesicles, endosomes, and lysosomes. (C) E6BSA-FITC is present along with FM4-64 fluorescent puncta in $\mathrm{N}-38$ neurons (image $\mathrm{C}$ is a close-up taken from a 100× image; $\mathrm{B}, \mathrm{C}$ are $100 \times$ images). BF: Bright field, CB: cell body.

in any given direction. A few puncta moved laterally adjacent to the plasma membrane with a directed motion as if moving along a defined path, traveling an average of $1,314 \pm 351 \mathrm{~nm}$. These puncta would occasionally halt and resume motion at random intervals. A few others, after stopping for several seconds, would move over long distances with dimming fluorescence intensity before disappearing (average time to disappear was $11.87 \pm 0.519 \mathrm{sec}$ ). Taken together, we called these traveling puncta "Type 1 Puncta." An example vesicle path is shown in Figure 4A. Other fluorescent E6BSA-FITC puncta, designated "Type 2 Puncta," would rapidly appear (increase in fluorescence intensity over $7.94 \pm 1.403 \mathrm{sec}$ on average) out of the dark interior of the cell beyond the evanescent illumination field, linger without movement in any favored direction (moving an average of $311.8 \pm 64.57 \mathrm{~nm}$ ) before rapidly brightening considerably and then quickly vanishing (Figure 4B). Type 1 puncta were significantly more mobile than Type 2 or stationary puncta (Figure $4 \mathrm{C}$; ANOVA $\mathrm{p}<0.0001, \mathrm{~F}_{(2,22)}=12.23$ ). However, we found that if the puncta moved at all, they moved with a consistent velocity, as can be seen for Type 1 and 2 puncta, compared with the stationary puncta group (Figure 4D).

Because many Type 1 puncta slowly dim as they move over time, implying movement up into the cell out of the evanescent field, we interpret these puncta as endocytotic vesicle trafficking (Figure 4A). Type 2 puncta, with their characteristic sudden brightening and rapid disappearance (Figure 4B), are consistent with vesicles fusing with the plasma membrane in exocytotic events. Furthermore, it is well known that FITC fluorescence is $\mathrm{pH}$ sensitive (FITC has a $\mathrm{pKa}$ 6.4) and fluorescence intensity is significantly increased at $\mathrm{pH} 7$ or above. Exocytotic vesicles are often acidic, with a $\mathrm{pH} 5.5-6$, which would cause a dimming of the fluorescence observed, until exocytosis occurs, wherein the $\mathrm{pH}$ the FITC experienced would rapidly increase to extracellular $\mathrm{pH}$ level (7.3 in these experiments), resulting in a sudden brightening of fluorescence before the released molecules diffuse away. Together, these observations are consistent with reports of vesicle trafficking, endocytosis, and exocytosis in other experimental models [see for example 21].

\section{Discussion}

In the present study, we investigated the regulation of plasma membrane ER trafficking using live N-38 neurons and TIRFM to trace the path of estradiol as it bound to ERs and rapidly traveled to and from the cell surface in real time. Using the combination of live cells and real-time imaging has permitted us to maintain cellular integrity and allowed us to obtain detailed information about the time scale and spatial distribution of ERs after activation. We found that: 1) E6BSA-FITC binds to the plasma membrane of N-38 neurons and is then internalized, 2) bound E6BSA-FITC moves in distinct patterns on and near the cell surface consistent with endocytotic and exocytotic events, and 3) E6BSA-FITC and GFP-ER $\alpha$ are associated with FM4-64 and LysoTracker positive endosomes, vesicles, and lysosomes. These results indicate that membrane-initiated ER $\alpha$ activation in live N-38 neurons causes rapid trafficking of the receptor at the cell surface. This is consistent with our previous studies using cell surface biotinylation where we found that estradiol or E6BSA treatment elevated full-length $\mathrm{ER} \alpha$ and $\mathrm{ER} \alpha \Delta 4$ protein levels on the plasma membrane of primary hypothalamic and N-38 neurons $[4,14]$.

Our findings suggest ERs are trafficked at the plasma membrane of N-38 neurons by a mechanism associated with vesicle trafficking (Figure 2C). Using TIRFM we observed E6BSA-FITC present on the plasma membrane and associated with endosomes, vesicles, and lysosomes. Pre-treatment with ER antagonist ICI prevented E6BSAFITC from binding, possibly by down-regulating plasma membrane ERs [39]. Using surface biotinylation we previously found that ERa trafficking is blocked by ICI or with the protein kinase $\mathrm{C}$ pathway inhibitor bisindolylmaleimide in N-38 neurons [4]. Membraneimpermeant estradiol compounds are known to be internalized several minutes after treatment ( $>5-30 \mathrm{~min}$ ) and the ligands have been observed to be bound to subcellular organelle membranes in different cell types [40-43]. Similar to the observations made by other investigators, we do not observe a significant level of BSA-FITC binding to the plasma membrane and internalization $[44,45]$. Our data suggest that plasma membrane ERs are internalized after ligand binding by endocytosis. In support, it was shown that when E6BSA-FITC treated primary cortical neurons were co-labeled with GM1, a lipid raft marker and endocytosis-indicator, we found the ER ligand and lipid raft indicator associated on the cell surface and with endosomes [46].

Several biochemical and electrophysiological studies provide evidence that membrane-initiated estradiol signaling regulates female reproductive behaviors by controlling the trafficking neurotransmitter receptors in hypothalamic neurons [2,10]. Estradiol triggered receptor trafficking may also be involved in hippocampal plasticity [46-48], and estradiol treatment has been reported to induce pit formation on the membranes of hypothalamic neurons [49,50]. Such pits may represent the fission of endocytotic pits responsible for receptor internalization and fusion exocytotic vesicles delivering $\mathrm{ERa}$ to the membrane [42,49-51]. Moreover, membrane ERa is associated with caveolae [52] and clathrin-coated vesicles $[41,53]$. Both these cellular membrane structures are lipid rich microdomains that cluster signal transduction machinery and proteins involved in membrane trafficking. Prior studies support the presence of both ERa and ER $\beta$ on the plasma membrane and the membranes of vesicles, endosomes, mitochondria, lysosomes, and microsomes [46,54-58]. Evidence also exists that shows the presence of other ER-like proteins present on the plasma membranes and organelles of neurons [6]. Our data, visualized in live $\mathrm{N}-38$ neurons in real-time, further supports this view.

The movement of Type 1, Type 2, and stationary puncta is consistent with vesicle motion observed in other cell types with TIRFM [59], which supports the idea that E6BSA-FITC is taken up and 


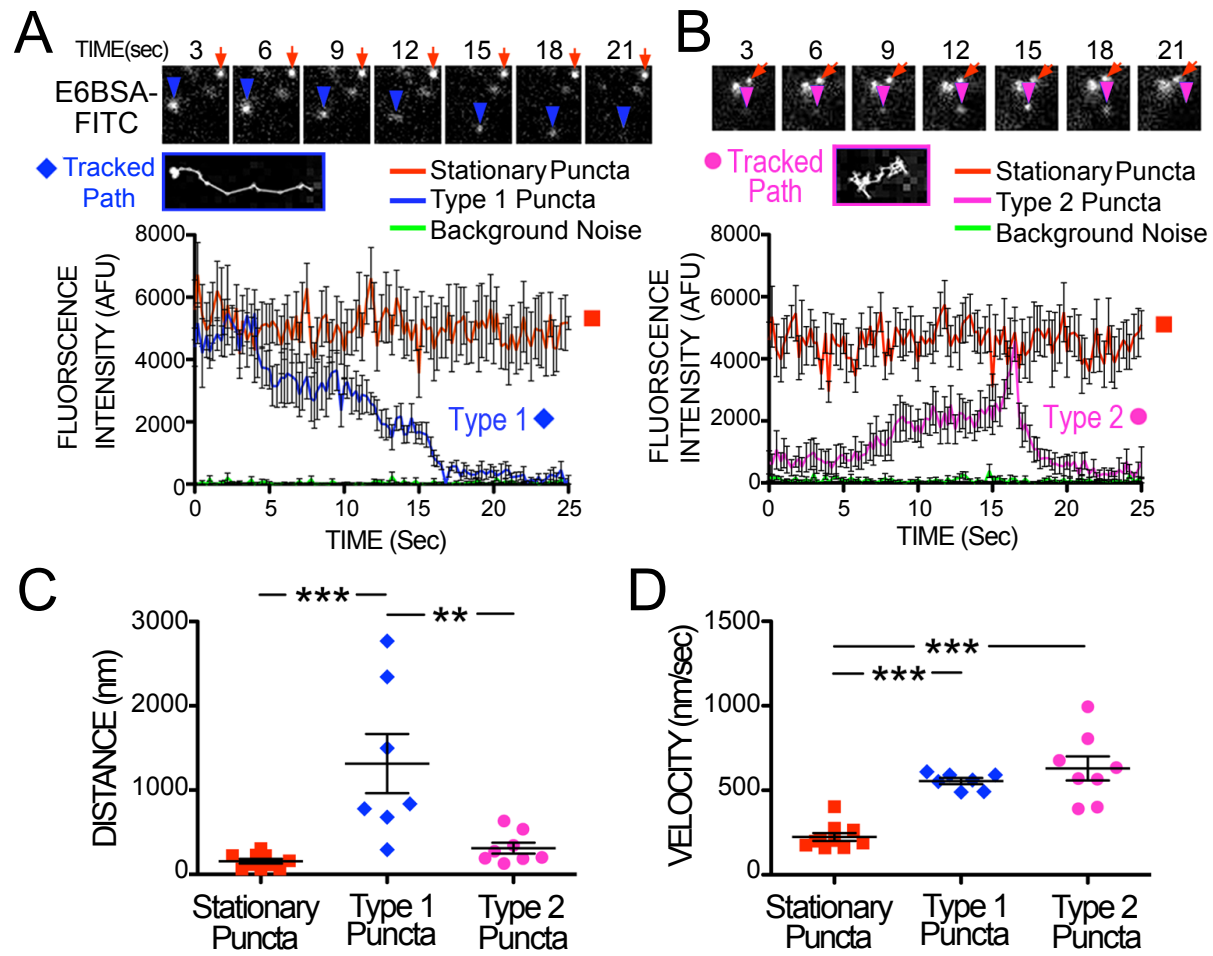

Figure 4: Fluorescent E6BSA-FITC is trafficked in and out of the plasma membrane. (A, B) Frame-by-frame tracking (4 frames per sec) of E6BSA-FITC fluorescent puncta intensity and position revealed different types of fluorescent molecule movements. E6BSA-FITC puncta were mostly stationary (orange squares), however a few initially stationary fluorescent puncta (A; Type 1; blue diamonds) suddenly moved long distances along a directed path (also see Tracked Path inset) only to dim away gradually. (B) Other puncta (Type 2; magenta circles) rapidly appeared, moved randomly a short distance (also see Tracked Path inset) and then rapidly ( 2.5 sec) disappeared. AFU: Arbitrary fluorescence units. Distance and speed of puncta movement is quantified in panels (C) and (D).

released from vesicular and/or endosomal compartments. Similarly, trafficking of ERa at the cell surface has also been observed in primary hypothalamic neurons [14]. Interestingly, previous work in our lab [60], reported that vesicle motion characteristics depended on the age of the vesicles in adrenal chromaffin cells, with the older vesicles moving greater distances, but with a lower likelihood of exocytosis, than newer vesicles. This is also consistent with our observations, where we see the type 2 puncta move shorter distances than the type 1 , and are observed to exocytose, although we cannot comment about the relative ages of type 1 and 2 puncta. Furthermore, fluorescence patterns of type 1 and 2 puncta over time are consistent with that reported in previous studies of exo- and endocytosis in other cell types [60-64].

It is not completely clear how ERa is regulated at the cell surface. The association of GFP-ERa with LysoTracker positive vesicles suggests the receptor is associated with lysosomes. Like other membrane receptors, plasma membrane ERa is likely regulated by a cellular mechanism associated with receptor trafficking. While definitive evidence has yet to be reported several questions remain such as how are plasma membrane ERs regulated at the plasma membrane. Evidence shows that portions of ER $\alpha$ protein structure biophysically interact with lipids in the bilayer and it potentially forms a transmembrane spanning region [17,65]. Cell surface biotinylation experiments have also made this point evident. The ability to biotinylate ERa protein suggests a portion of the receptor protein is exposed to the extracellular space $[12,46,66,67]$. In neurons and cell lines palmitoylation has been shown to regulate membrane localization, ERa degradation and transcriptional activity [68-70]. We have found that ER $\alpha$ forms a signaling complex with $\beta$-arrestin1, an adaptor protein involved in receptor desensitization and down- regulation [46]. One sign of this pathway is the activation of GRKs and receptor phosphorylation. We have shown the activation of GRK2 by estradiol [46] and a recent study has reported that phosphorylation of active $\mathrm{ER} \alpha$ on serine residue 118 and tyrosine residue 537 results in the receptor being directed toward degradation $[69,71]$. Existing evidence and that presented here suggest that estradiol regulates the ERa plasma membrane levels by modulating the receptors' expression through trafficking and down-regulation.

In summary, using TIRFM we demonstrated in real-time the trafficking of E6BSA-FITC in N-38 neurons. Our data provides further evidence that membrane-initiated ERa signaling present in $\mathrm{N}-38$ neurons is regulated by a similar mechanism present in different neuronal populations. Additionally, we have shown that the use of TIRFM in combination with N-38 neurons provides a model system to examine real-time membrane-initiated receptor trafficking at the cellular level. We hypothesize that membrane-initiated estradiol signaling involves the fusing of plasma membrane and formation of ERa-containing endosomes.

\section{Acknowledgment}

These studies were supported by a NIH grant to RD (NS079064).

\section{References}

1. Wilson ME, Westberry JM (2009) Regulation of oestrogen receptor gene expression: new insights and novel mechanisms. J Neuroendocrinol 21: 238242.

2. Micevych $P$, Dominguez $R$ (2009) Membrane estradiol signaling in the brain Front Neuroendocrinol 30: 315-327. 
Citation: Kisler K, Chow RH, Dominguez R (2013) Fluorescently-Labeled Estradiol Internalization and Membrane Trafficking in Live N-38 Neuronal Cells Visualized with Total Internal Reflection Fluorescence Microscopy. J Steroids Hormon Sci S12: 002. doi:10.4172/2157-7536.S12-002

Page 6 of 7

3. Micevych PE, Kelly MJ (2012) Membrane estrogen receptor regulation of hypothalamic function. Neuroendocrinology 96: 103-110.

4. Dominguez R, Dewing $P$, Kuo J, Micevych $P$ (2013) Membrane-initiated estradiol signaling in immortalized hypothalamic N-38 neurons. Steroids .

5. Heldring N, Pike A, Andersson S, Matthews J, Cheng G, et al. (2007) Estrogen receptors: how do they signal and what are their targets. Physiol Rev 87: 905931.

6. Levin ER (2009) Plasma membrane estrogen receptors. Trends Endocrino Metab 20: 477-482.

7. Minami T, Oomura Y, Nabekura J, Fukuda A (1990) 17 beta-estradio depolarization of hypothalamic neurons is mediated by cyclic AMP. Brain Res 519: 301-307.

8. Nabekura J, Oomura Y, Minami T, Mizuno Y, Fukuda A (1986) Mechanism of the rapid effect of 17 beta-estradiol on medial amygdala neurons. Science 233: 226-228.

9. Gu Q, Moss RL (1996) 17 beta-Estradiol potentiates kainate-induced currents via activation of the cAMP cascade. J Neurosci 16: 3620-3629.

10. Sinchak K, Micevych P (2003) Visualizing activation of opioid circuits by internalization of $G$ protein-coupled receptors. Mol Neurobiol 27: 197-222.

11. Gainetdinov RR, Premont RT, Bohn LM, Lefkowitz RJ, Caron MG (2004) Desensitization of $\mathrm{G}$ protein-coupled receptors and neuronal functions. Annu Rev Neurosci 27: 107-144.

12. Bondar G, Kuo J, Hamid N, Micevych P (2009) Estradiol-induced estrogen receptor-alpha trafficking. J Neurosci 29: 15323-15330.

13. Li L, Hisamoto K, Kim KH, Haynes MP, Bauer PM, et al. (2007) Variant estrogen receptor-c-Src molecular interdependence and c-Src structural requirements for endothelial NO synthase activation. Proc Natl Acad Sci U S A 104: 1646816473.

14. Dominguez $R$, Micevych $P$ (2010) Estradiol rapidly regulates membrane estrogen receptor alpha levels in hypothalamic neurons. J Neurosci 30: 1258912596

15. Titolo D, Cai F, Belsham DD (2006) Coordinate regulation of neuropeptide $Y$ and agouti-related peptide gene expression by estrogen depends on the ratio of estrogen receptor (ER) alpha to ERbeta in clonal hypothalamic neurons. Mol Endocrinol 20: 2080-2092.

16. Belsham DD, Cai F, Cui H, Smukler SR, Salapatek AM, et al. (2004) Generation of a phenotypic array of hypothalamic neuronal cell models to study complex neuroendocrine disorders. Endocrinology 145: 393-400.

17. Kim KH, Toomre D, Bender JR (2011) Splice isoform estrogen receptors as integral transmembrane proteins. Mol Biol Cell 22: 4415-4423.

18. Chen D, Patrick JW (1997) The alpha-bungarotoxin-binding nicotinic acetylcholine receptor from rat brain contains only the alpha7 subunit. J Biol Chem 272: 24024-24029.

19. Axelrod D, Thompson NL, Burghardt TP (1983) Total internal inflection fluorescent microscopy. J Microsc 129: 19-28.

20. Wang MD, Axelrod D (1994) Time-lapse total internal reflection fluorescence video of acetylcholine receptor cluster formation on myotubes. Developmental dynamics : an official publication of the American Association of Anatomists 201: $29-40$.

21. Steyer JA, Almers W (2001) A real-time view of life within $100 \mathrm{~nm}$ of the plasma membrane. Nat Rev Mol Cell Biol 2: 268-275.

22. Toomre D, Manstein DJ (2001) Lighting up the cell surface with evanescent wave microscopy. Trends Cell Biol 11: 298-303.

23. Axelrod D (2008) Chapter 7: Total internal reflection fluorescence microscopy. Methods Cell Biol 89: 169-221.

24. Taguchi Y, Koslowski M, Bodenner DL (2004) Binding of estrogen receptor with estrogen conjugated to bovine serum albumin (BSA). Nucl Recept 2: 5.

25. Stenoien DL, Mancini MG, Patel K, Allegretto EA, Smith CL, et al. (2000) Subnuclear trafficking of estrogen receptor-alpha and steroid receptor coactivator-1. Mol Endocrinol 14: 518-534.

26. Michael DJ, Ritzel RA, Haataja L, Chow RH (2006) Pancreatic beta-cells secrete insulin in fast- and slow-release forms. Diabetes 55:600-607.
27. Meijering E, Dzyubachyk O, Smal I (2012) Methods for Cell and Particle Tracking. Methods in Enzymology 504: 183-200.

28. Sako Y, Minoghchi S, Yanagida T (2000) Single-molecule imaging of EGFR signalling on the surface of living cells. Nat Cell Biol 2: 168-172.

29. Grimes ML, Zhou J, Beattie EC, Yuen EC, Hall DE, et al. (1996) Endocytosis of activated TrkA: evidence that nerve growth factor induces formation of signaling endosomes. J Neurosci 16: 7950-7964.

30. Berthois $Y$, Pourreau-Schneider N, Gandilhon P, Mittre H, Tubiana N, et al. (1986) Estradiol membrane binding sites on human breast cancer cell lines. Use of a fluorescent estradiol conjugate to demonstrate plasma membrane binding systems. J steroid Biochem 25: 963-972.

31. Razandi M, Pedram A, Merchenthaler I, Greene GL, Levin ER (2004) Plasma membrane estrogen receptors exist and functions as dimers. Mol Endocrinol 18: $2854-2865$

32. De Goeij AF, van Zeeland JK, Beek CJ, Bosman FT (1986) Steroid-bovine serum albumin conjugates: molecular characterization and their interaction with androgen and estrogen receptors. J Steroid Biochem 24: 1017-1031.

33. Stevis PE, Deecher DC, Suhadolnik L, Mallis LM, Frail DE (1999) Differential effects of estradiol and estradiol-BSA conjugates. Endocrinology 140: 54555458

34. Wilkinson RS, Lin MY (2004) Endocytosis and synaptic plasticity: might the tail wag the dog? Trends Neurosci 27: 171-174.

35. Betz WJ, Mao F, Bewick GS (1992) Activity-dependent fluorescent staining and destaining of living vertebrate motor nerve terminals. J Neurosci 12: 363-375.

36. Betz WJ, Bewick GS (1992) Optical analysis of synaptic vesicle recycling at the frog neuromuscular junction. Science 255: 200-203.

37. Taraska JW, Almers W (2004) Bilayers merge even when exocytosis is transient. Proc Natl Acad Sci U S A 101: 8780-8785

38. Jiang LW, Maher VM, McCormick JJ, Schindler M (1990) Alkalinization of the lysosomes is correlated with ras transformation of murine and human fibroblasts. J Biol Chem 265: 4775-4777.

39. Dudley MW, Sheeler CQ, Wang H, Khan S (2000) Activation of the human estrogen receptor by the antiestrogens ICI 182,780 and tamoxifen in yeast genetic systems: implications for their mechanism of action. Proc Natl Acad Sci U S A 97: 3696-3701.

40. Karthikeyan N, Thampan RV (1996) Plasma membrane is the primary site of localization of the nonactivated estrogen receptor in the goat uterus: hormone binding causes receptor internalization. Arch Biochem Biophys 325: 47-57.

41. Moats RK 2nd, Ramirez VD (2000) Electron microscopic visualization of membrane-mediated uptake and translocation of estrogen-BSA:colloidal gold by hep $\mathrm{G} 2$ cells. J Endocrinol 166: 631-647.

2. Moats RK 2nd, Ramirez VD (1998) Rapid uptake and binding of estradiol17beta-6-(O-carboxymethyl)oxime:125I-labeled BSA by female rat liver. Bio Reprod 58: 531-538

43. Marin R, Guerra B, Alonso R, Ramírez CM, Díaz M (2005) Estrogen activates classical and alternative mechanisms to orchestrate neuroprotection. Curr Neurovasc Res 2: 287-301.

44. Nenci I, Marchetti E, Marzola A, Fabris G (1981) Affinity cytochemistry visualizes specific estrogen binding sites on the plasma membrane of breast cancer cells. J Steroid Biochem 14: 1139-1146.

45. Wu TW, Chen S, Brinton RD (2011) Membrane estrogen receptors mediate calcium signaling and MAP kinase activation in individual hippocampal neurons. Brain Res 1379: 34-43.

46. Dominguez R, Hu E, Zhou M, Baudry M (2009) 17beta-estradiol-mediated neuroprotection and ERK activation require a pertussis toxin-sensitive mechanism involving GRK2 and beta-arrestin-1. J Neurosci 29: 4228-4238.

47. Zadran S, Qin Q, Bi X, Zadran H, Kim Y, et al. (2009) 17-Beta-estradio increases neuronal excitability through MAP kinase-induced calpain activation. Proc Natl Acad Sci U S A 106: 21936-21941.

48. Aguirre C, Jayaraman A, Pike C, Baudry M (2010) Progesterone inhibits estrogen-mediated neuroprotection against excitotoxicity by down-regulating estrogen receptor- $\hat{I}^{2}$. J Neurochem 115: 1277-1287.

49. Garcia-Segura LM, Olmos G, Tranque P, Naftolin F (1987) Rapid effects of 
Citation: Kisler K, Chow RH, Dominguez R (2013) Fluorescently-Labeled Estradiol Internalization and Membrane Trafficking in Live N-38 Neuronal Cells Visualized with Total Internal Reflection Fluorescence Microscopy. J Steroids Hormon Sci S12: 002. doi:10.4172/2157-7536.S12-002

gonadal steroids upon hypothalamic neuronal membrane ultrastructure. $\mathrm{J}$ Steroid Biochem 27: 615-623.

50. Olmos G, Aguilera P, Tranque P, Naftolin F, Garcia-Segura LM (1987) Estrogen-induced synaptic remodelling in adult rat brain is accompanied by the reorganization of neuronal membranes. Brain Res 425: 57-64

51. Párducz A, Szilágyi T, Hoyk S, Naftolin F, Garcia-Segura LM (1996) Neuroplastic changes in the hypothalamic arcuate nucleus: the estradiol effect is accompanied by increased exoendocytotic activity of neuronal membranes. Cell Mol Neurobiol 16: 259-269.

52. Luoma JI, Boulware MI, Mermelstein PG (2008) Caveolin proteins and estrogen signaling in the brain. Mol Cell Endocrinol 290: 8-13.

53. Sreeja S, Thampan RV (2004) Proteins which mediate the nuclear entry of goat uterine non activated estrogen receptor (naER) following naER internalization from the plasma membrane. Mol Cell Biochem 259: 141-148.

54. Clarke CH, Norfleet AM, Clarke MS, Watson CS, Cunningham KA, et al. (2000) Perimembrane localization of the estrogen receptor alpha protein in neuronal processes of cultured hippocampal neurons. Neuroendocrinology 71: 34-42.

55. Blaustein JD, Lehman MN, Turcotte JC, Greene G (1992) Estrogen receptors in dendrites and axon terminals in the guinea pig hypothalamus. Endocrinology 131: $281-290$.

56. Revankar CM, Cimino DF, Sklar LA, Arterburn JB, Prossnitz ER (2005) A transmembrane intracellular estrogen receptor mediates rapid cell signaling. Science 307: 1625-1630.

57. Romeo RD, McCarthy JB, Wang A, Milner TA, McEwen BS (2005) Sex differences in hippocampal estradiol-induced $\mathrm{N}$-methyl-D-aspartic acid binding and ultrastructural localization of estrogen receptor-alpha. Neuroendocrinology 81: 391-399.

58. Hart SA, Snyder MA, Smejkalova T, Woolley CS (2007) Estrogen mobilizes a subset of estrogen receptor-alpha-immunoreactive vesicles in inhibitory presynaptic boutons in hippocampal CA1. J Neurosci 27: 2102-2111.

59. Huet S, Karatekin E, Tran VS, Fanget I, Cribier S, et al. (2006) Analysis of transient behavior in complex trajectories: application to secretory vesicle dynamics. Biophys J 91: 3542-3559.

60. Duncan RR, Greaves J, Wiegand UK, Matskevich I, Bodammer G, et al. (2003) Functional and spatial segregation of secretory vesicle pools according to vesicle age. Nature 422: 176-180.
61. Toomre D, Steyer JA, Keller P, Almers W, Simons K (2000) Fusion of constitutive membrane traffic with the cell surface observed by evanescent wave microscopy. J Cell Biol 149: 33-40.

62. Allersma MW, Wang L, Axelrod D, Holz RW (2004) Visualization of regulated exocytosis with a granule-membrane probe using total internal reflection microscopy. Mol Biol Cell 15: 4658-4668.

63. Merrifield CJ, Feldman ME, Wan L, Almers W (2002) Imaging actin and dynamin recruitment during invagination of single clathrin-coated pits. Nat Cell Biol 4: 691-698.

64. Rappoport JZ, Kemal S, Benmerah A, Simon SM (2006) Dynamics of clathrin and adaptor proteins during endocytosis. Am J Physiol Cell Physiol 291 C1072-1081.

65. Byrne C, Khemtémourian L, Pelekanou V, Kampa M, Leclercq G, et al. (2012) ERI $\pm 17 p$, a peptide reproducing the hinge region of the estrogen receptor \pm associates to biological membranes: A biophysical approach. Steroids 77 979-987.

66. Gorosito SV, Lorenzo AG, Cambiasso MJ (2008) Estrogen receptor alph is expressed on the cell-surface of embryonic hypothalamic neurons. Neuroscience 154: 1173-1177.

67. Li L, Haynes MP, Bender JR (2003) Plasma membrane localization and function of the estrogen receptor alpha variant (ER46) in human endothelial cells. Proc Natl Acad Sci U S A 100: 4807-4812.

68. Acconcia F, Ascenzi P, Bocedi A, Spisni E, Tomasi V, et al. (2005) Palmitoylation-dependent estrogen receptor alpha membrane localization: regulation by 17 beta-estradiol. Mol Biol Cell 16: 231-237.

69. La Rosa P, Pesiri V, Leclercq G, Marino M, Acconcia F (2012) Palmitoylation regulates $17 \hat{I}^{2}$-estradiol-induced estrogen receptor-î \pm degradation and transcriptional activity. Mol Endocrinol 26: 762-774.

70. Tabatadze N, Smejkalova T, Woolley CS (2013) Distribution and posttranslational modification of synaptic ERI \pm in the adult female rat hippocampus. Endocrinology 154: 819-830.

71. Sun J, Zhou W, Kaliappan K, Nawaz Z, Slingerland JM (2012) ERÎ \pm phosphorylation at $\mathrm{Y} 537$ by Src triggers E6-AP-ER \pm binding, ERÎ \pm ubiquitylation, promoter occupancy, and target gene expression. Mol Endocrinol 26: $1567-1577$.
This article was originally published in a special issue, Steroid Hormone Metabolism handled by Editor. Dr. Carin Wittnich, University of

Toronto, Canada 